\title{
An Overview of Robotics and Autonomous Systems for Harsh Environments
}

\author{
Cuebong Wong ${ }^{1}$, Erfu Yang ${ }^{1}$, Xiu-Tian Yan $^{1}$ and Dongbing $\mathrm{Gu}^{2}$
}

\author{
${ }^{1}$ Space Mechatronic Systems Technology Laboratory \\ Strathclyde Space Institute, Department of Design, \\ Manufacture and Engineering ManagementUniversity of \\ Strathclyde, Glasgow G1 1XJ, UK \\ Emails: \{Cuebong.wong, erfu.yang, x.yan\}@strath.ac.uk
}

\author{
${ }^{2}$ School of Computer Science and Electronic Engineering \\ University of Essex \\ Wivenhoe Park, Colchester CO4 3SQ, UK \\ Email:dgu@essex.ac.uk
}

\begin{abstract}
Across a wide range of industries and applications, robotics and autonomous systems can fulfil the crucial and challenging tasks such as inspection, exploration, monitoring, drilling, sampling and mapping in areas of scientific discovery, disaster prevention, human rescue and infrastructure management, etc. However, in many situations, the associated environment is either too dangerous or inaccessible to humans. Hence, a wide range of robots have been developed and deployed to replace or aid humans in these activities. A look at these harsh environment applications of robotics demonstrate the diversity of technologies developed. This paper reviews some key application areas of robotics that involve interactions with harsh environments (such as search and rescue, space exploration, and deep-sea operations), gives an overview of the developed technologies and provides a discussion of the key trends and future directions common to many of these areas.
\end{abstract}

Keywords-robotics, autonomous systems, harsh environments, human-robot interaction, tele-operation

\section{INTRODUCTION}

Without any doubt, robotics and autonomous systems (RAS) are playing an increasingly important role across a wide range of areas. No longer are robots only found exclusively in manufacturing plants, but they have been making their way into widespread areas such as clinical centers, transportation, restaurants, military sites and domestic homes. Indeed, this growth will continue rapidly into the future, where autonomy and smart systems will integrate into even more aspects of our world. The development of RAS was inspired by a number of key benefits that could be derived from these systems. They offer possibilities not achievable by humans alone; they can perform repeated complex actions without fatigue; they can be deployed in place of humans for activities that are dangerous; they are, in general, efficient machines able to operate at high speeds and in some circumstances they are a cost-effective solution to problems.

For these reasons, much research efforts have been directed to the development of RAS for more challenging but rewarding applications. For example, the deployment of the Curiosity Rover in 2011 to go where no man has gone and explore the surface of Mar was a key milestone that showcases just some of the possibilities that may be realized through robotic technology. However, operating in these harsh environments bring additional challenges that cannot be resolved using traditional RAS solutions. Major advancements in both hardware and software have been found to be necessary to realize effective systems that can operate successfully for such applications. There is thus a growing demand for smarter, more adaptive and more autonomous robots across many applications. The recent emergence of the self-driving car is a noteworthy example of how such technology is being integrated into our lives to enhance human wellbeing. Through the implementation of state-of-the-art sensor technology and the development of data fusion and machine learning techniques, these smart cars are capable of making safe driving decisions on structured roads debatably as well as humans. Collaboration robots too have recently appeared in various environments, such as manufacturing, search and rescue and healthcare. Through this human-robot interaction (HRI), more complicated tasks can be achieved with greater success in highly challenging environments. Indeed, aspects relating to safety are a key priority for these applications.

There is no doubt that RAS technologies will continue to make its way into harsh environments, offering increasing benefits to a multitude of activities across a vast range of industries and sectors. In view of this rapid growth, this paper gathers together a representative selection of literature to provide an overview of the current state of robotics for applications in harsh environments. More precisely, we survey a range of robotic technologies developed for a variety of different harsh environment applications, such as search and rescue, planetary exploration and deep sea operations, and then we provide a discussion of key research trends and future directions.

This paper is arranged as follows. Section II provides a general definition of harsh environments and describes the applications of the RAS considered in this paper, while the literature survey is given in section III. Section IV gives a discussion of the findings from the perspective of the authors. 


\section{RESEARCH NEEDS AND CHALLENGES}

In this paper, harsh environments are defined as those which are unknown, unstructured, dynamic, cluttered, hazardous and/or limited in resources (such as the availability of communications, GPS and visibility). Indeed, a wide range of application areas fall under this definition and are representative problems of challenging real-world applications of robotics. In this section, we discuss a number of robotic application areas where robots must operate in some form of harsh environment. These will be expanded upon in section III, where robots used in these areas are reviewed.

Space robotics is commonly associated to the interactions with harsh environments. Orbital, planetary and asteroid robotics must operate in a mostly unexplored environment subject to extreme temperatures, high levels of radiation, limited communications and lack of GPS, among many other difficulties. Surfaces on extraterrestrial bodies are also challenging to navigate due to the rocky and sandy nature of the terrain. Most (if not all) sustained damage are impossible to fix due to inaccessibility of space, and tele-operation is often inefficient as a result of the long distances between the operator and the robot (in cases where robots are deployed away from the operator). These are problems that are still currently being addressed despite several successes with the exploration of Mars through Curiosity and its predecessors [1].

We do not need to look as far as space, however, to encounter problems from harsh environments. Many industrial activities within the energy sector benefit from the deployment of robotic systems. For example, the decommissioning of large facilities, such as nuclear power plants, is much more suited to robots due to the danger posed by residual radiation to human personnel. Similarly, the aspects of safety are key drivers for the deployment of robotic systems in the oil and gas industry. Past disasters such as the oil spill in the Gulf of Mexico have taught the importance of early detection of faults in offshore infrastructure that are hard to access without the use of mobile robots. Robots are therefore required to access small pipe-like areas often filled with fluids. The harshness of the environment is further elevated by the potentially explosive atmospheres commonly found at oil and gas sites.

Likewise, deep-sea operations are another application area which benefits greatly from the development of RAS. To date, most of these activities have been performed with human divers, which is both a costly and high-risk solution. The deployment of underwater vehicles enables the more efficient completion of tasks while minimizing danger to human workers. However, deep-sea conditions vary drastically over time; currents cause robots to drift and the presence of water blocks the availability of GPS signals. Hence localization is a difficult problem to solve for deep-sea applications. Furthermore, maneuvering in water cannot be achieved with simple wheel mechanisms and the risk of water damage must be considered as well.

In search and rescue applications, robots are required to work efficiently in environments with unknown and varying topological landscapes. Here, robots play critical roles to support rescue activities, such as mapping the environment, monitoring the local environmental parameters to assess the safety conditions and searching for survivors in areas that are dangerous or inaccessible to human rescue teams. These are highly complex tasks due to the difficulty of traversing local (possibly damaged) terrain, low visibility, interruptions to communications and information transmission lag. The conditions in which robots must operate in can vary significantly depending on whether it is deployed indoors or outdoors. For example, robots used to assist firefighters in indoor firefighting must be capable of navigating through cluttered and confined environments while working collaboratively with human team members [2]. For search and rescue in outdoor environments, localization, mapping and target identification becomes much more complex due to the vast scale of the environment in which the robot operates. These are challenging problems given the constraints on limited onboard resources for mobile robotic systems.

Polar exploration is another area in which the navigation of mobile robots is challenging but necessary. The exploration of Polar Regions (including polar ice sheets and glaciers) are important to scientific research. For example, data gathered from these regions enable scientists to better understand how the world works, as well as forecasting key trends such as weather and climate changes. Yet, much of these regions are unreachable for humans due to their extreme environmental conditions. Even for robotic systems, it is a challenge to navigate the uneven, icy and snowy surfaces with very few features that can be used as reference landmarks for the robot.

Perhaps less commonly associated with harsh environments are robots used in the medical field. Surgical in-vivo robots offer assistance to many surgical tasks and provide valuable visual feedback of internal parts of the body to both on-site and remote surgeons. The benefits provided by the use of these robots greatly enhance the development of minimally invasive surgery, which has become increasingly popular due to associated faster recovery times and smaller incisions required. But the human body itself is a harsh environment for such robots, where spaces are highly confined and surrounding tissues are delicate. Furthermore, these spaces are complex in form and therefore pose a certain level of difficulty for navigating robots through.

While we have not exhaustively described or listed all types of harsh environments in which RAS technologies can be applied to, we can begin to identify a number of commonalities and traits across the aforementioned types of harsh environments. Certainly, many harsh environments share similar properties that can be resolved using techniques already developed for another application.

\section{Key Technology DeVelopment ReVIEW}

\section{A. Oil and Gas}

Within the oil and gas industry, robotic systems have begun to appear more commonly both in onshore and offshore 
applications for numerous reasons. Among these, health, safety and environment concerns are the key drivers for the deployment of RAS technologies in this field. As easily accessible oil and gas reservoirs are becoming rapidly depleted, it has become necessary to extract such resources from more remote and harsher environments to meet the growing global demand for fossil fuels. Onshore, these sites may be located in arctic or hot desert environments with extreme temperatures and severe weather conditions, while offshore rigs stretch down into the deep sea. With the integrity of infrastructure being a key concern due to the scale of damages that may be caused from failures, regular inspection of these structures are a necessity. Sending humans to perform these inspections directly is expensive and poses a serious risk to their health and safety. Hence there are many opportunities for robotic applications in the oil and gas industry.

RAS technologies developed for oil and gas facilities come in multiple forms. Mobile robots are used to perform a broad range of activities, such as tank inspection, pipeline inspection, monitoring and surveillance, valve/lever operation and maintenance. In [3] a mobile robot was developed to perform a number of routine tasks at a refinery which otherwise would have been performed by humans. Instead, a human operator assumes a supervisory role, providing the robot with individual commands in teleoperation mode, or high-level task goals for semi-autonomous navigation mode. In the latter case, the robot handles all low-level commands such as path planning, collision avoidance and localization while the human operator is responsible for cognitive decision-making. This robot (and those similar to it [4]) is equipped with a manipulator, gas detectors, acoustic sensors, thermal imaging and video cameras, which enables it to perform a flexible range of tasks. This is particularly essential for handling unforeseen emergencies that require high level of adaptability to various scenarios.

In [5], the authors presented a review of in-pipe inspection robots (IPIRs) used to detect cracks, corrosion and other types of defects which can lead to pipe failure. These robots, equipped with non-destructive testing (NDT)-based sensors (such as eddy current and magnetic flux leakage probes etc.) are inserted internally into pipelines and propelled along the pipeline network. These robots can be further classified by their means of propulsion. For example, there are wheel-based, legged, screw-based, snake and pipeline inspection gauge type IPIRs. IPIRs may be manually-operated, semi-autonomous (requiring human input for decision making) and fully autonomous (requiring no external commands from the operator). However, due to the unproven reliability of fully autonomous operation, semi-autonomous control is still the preferred mode of operation. These robots are usually powered by a tether cable that leads out of the pipelines, which also carries data transmission and control signals to and from an operator system. This can impose certain restrictions to the robot due to additional friction forces and twisting of the cable. Hence tether-less robots have also recently been proposed ([6]). The development of tether-less IPIR is an ongoing challenge as a robust means of wireless power must be provided through the walls of the pipeline, and the limited internal space of the navigation space restricts resources available on-board the system.

Other technologies have also been deployed for the monitoring of pipeline integrity. In particular, wireless sensor networks (WSNs) [7] are a cost-effective and reliable way of detecting build-up of sand, pipe damage and fluid leakage, as well as acting as an anti-theft system. Originally these sensors were installed with wired connections. However, wires are prone to damage that would subsequently cause a break in the network. An alternative to this is the installation of batteries to these sensor networks to act as failsafe power in the event of broken wired connections. Truly wireless solutions which do not consist of any wiring across sensors rely on a sleep-wake cycle to conserve energy, whereby sensors are active for a few seconds before switching to an idle state for up to several minutes. Expanding on the idea of WSNs are robotic sensor networks. Here, wireless sensor nodes are carried by in-pipe robots and communicate with evenly spaced relay nodes across the pipeline infrastructure. These relay nodes relay information back to a single base station. Such a method enables more accurate and adaptable inspection strategies. Furthermore, the self-localization of the in-pipe robots are enhanced by relay nodes, which acts as beacons along the pipeline [8].

In [9], a tactile exploration method to mapping pipelines and similar structures is presented, which solely uses a manipulator with joint encoders mounted on a remotely operated underwater vehicle (ROV). With the ROV anchored onto pipelines, contact is maintained between the tip of the manipulator and the surface of interest, thus allowing the joint encoders to provide sufficient information to map its form in harsh situations where other sensors are inappropriate. For example, during severe leakages, escaping fluids obscure the vision of cameras, while high turbulence and mixture of fluids significantly impair the reliability of laser and sonar sensors. The authors' experimental results demonstrated the feasibility and robustness of this approach under various harsh environment conditions through simulated experiments.

Further inspection applications of RAS in oil and gas include the deployment of wall-climbing robots for tank inspection. Mostly used in teleoperation mode, these robots rely upon magnetism, vacuum suction or external attachment devices to grip onto the walls of tanks. Locomotion is achieved through a variety of means, such as wheels, legs or tracks, and continuous inspection is performed using on-board NDT sensors (such as visual cameras, ultrasonic sensors and acoustics) [5]. In cases where structures possess thin walls that are insufficient in strength to support heavy robots, a mother and child architecture has been proposed [10]. The "mother" robot, which provides adhesion and locomotion equipment, attaches onto stronger support structures such as a track or rail. The child robot, often a physically extended inspection device, then performs the inspection on the walls of the tank. Alternatively, unmanned aerial vehicles (UAVs) such as drones may also be used to perform inspection of tanks, as well as 
pipelines and refineries as a whole ([11], [12]). These robots are either manually controlled or flown in semi-autonomous mode, with an operator providing high level commands from a ground control station. As such, these systems rely heavily upon robust flight control techniques consisting of dead reckoning, inertial navigation, data fusion and tracking control aspects. Furthermore, UAVs may also be deployed for the exploration of oil and gas fields located in more remote and harsh environments not suitable for human exploration. Hence, much developmental work has been devoted to multi-UAV systems, where several robots must be coordinated flawlessly to cover a larger area more efficiently.

\section{B. Underwater/Deep Sea Robotics}

The applications of RAS technologies for underwater and deep sea activities are vast. One such application is the use of ROVs to replace divers for archaeological surveying. In [13], a method for using an ROV with multiple sensors and platforms for deep sea archaeological discovery was presented. This is a challenging task both in terms of controlling the ROV (due to depth and currents, as well as the dependence on a supporting surface vessel) and in terms of the identification and recording of archaeological specimens. The authors of the paper discuss how data obtained from one sensor may be used to plan subsequent tasks with other sensors such that an overall better resolution dataset may be obtained from the consolidation of information from multiple sensors. These sensors include optical-based devices such as light detection and ranging (LiDAR) and visual cameras, and acoustic sensors.

Similarly, ROVs have also been deployed for exploration activities in ice-covered waters. A preliminary survey of underwater robotic vehicles for under-ice operations was presented in [14], where various tested systems designed for both static ice and moving sea ice conditions were discussed. These systems were classified as tethered, untethered, hybridtethered (where the tethering cable is intended only for the transmission of information and supplies no power to the ROV), or glider (which uses a buoyancy engine and horizontal wings for propulsion). With the absence of GPS signals in these waters, localization of the robot was achieved by the use of acoustic beacons fixed onto ice along the intended path of the vehicle. An interesting discussion in this paper was the comparison between ship-based and through-ice methods of deploying ROVs into the waters. While deployment from a ship is dependent upon weather and ice conditions, through-ice deployment requires additional effort in the form of drilling/melting ice. Other example applications of ROV deployment include dam inspection (tethered onto a boat and fixed to the walls of the dam) [15] and long-term deep sea observation through the use of an underwater docking station for wireless charging [16].

The authors in [17] presented a maritime platform for search and rescue, which was submitted to the euRathlon 2015 competition. Here an unmanned surface vessel, ROAZ, was used cooperatively with a Mares autonomous underwater vehicle (AUV) to provide enhanced perception capabilities for both small and large scale rescue activities. The ROAZ system carried functionalities such as computer navigation and control, RADAR, thermographic and visible spectrum camera sensing, and autonomous mission control. It also provided the necessary resources for processing sensor data from the Mares vehicle. The AUV was primarily deployed for remote monitoring, survey and modelling tasks, and was propelled using two forward and two vertical thrusters. Collaboratively, this multirobot system was able to perform tasks such as underwater valve actuation, target detection, and underwater search, survey and mapping.

Most recently, other configurations of robotic systems have been developed for harsh underwater environments. For example, Tanaka et al. presented an underwater robot inspired by the concept of a quadcopter [18]. By using four rotor thrusters to counteract buoyancy forces, underwater flight control could be achieved. This is particularly useful for applications where the position of a robot must be fixed within harsh underwater environments. The four thrusters enable the robot to resist disturbances so that fixed point observations may be achieved. In Spring 2016, Ocean One, a humanoid robot with human-like manipulation skills, had also been successfully deployed for underwater discovery activities [19]. This robot possessed a higher level of intelligence as compared to traditional ROV systems and could operate with high levels of autonomy. Its human-like features further opened up the possibilities of HRI in underwater operations, and was connected to a human operator on the surface who could take over for high level guidance when needed. Communications and recharging on Ocean One's on-board battery was achieved through a tethered relay station, thus allowing multiple robots to operate at any time. This work was a clear demonstration of the advances made in RAS technology and encapsulates concepts of HRI and multi-robot systems.

\section{Space Exploration}

Outer space is undoubtedly the most vast, unreachable and harsh environment for humans to explore. The use of RAS technologies is therefore essential to space exploration and scientific discovery. Achievements in this field can be broadly divided into three classes: orbital robotics, asteroid robotics and planetary robotics [20]. Orbital robotics typically consist of free-flying robots for purposes such as assembly of space structures, space debris rescue, unmanned orbital operations and routine satellite maintenance and servicing tasks. To achieve these goals, the robot must possess retrieval and docking functionalities typically provided by at least one manipulator arm. For the development of asteroid robotics, a major challenge is introduced by the micro-gravity environment. Locomotion becomes a more complicated task as there is insufficient wheel traction to permit the use of basic wheel-based locomotion designs. Existing solutions to this problem include the use of an internal flywheel to create a hopping and tumbling motion across the surface; wheels attached to swingable struts to provide the required traction; and articulated robots that grasp and walk across the surface (inspired by the concept of rock climbing). Often asteroid 
robots are required to collect samples from these extraterrestrial bodies. For example, [21] reviewed robotic mining concepts for outer space, where RAS technology was used to harvest valuable resources available only in extraterrestrial surfaces.

A number of planetary robots have been deployed to Mars with notable success. These systems are tasked objectives such as sampling local soil and rocks, mapping the environment, capturing images of key landmarks, and monitoring local environment conditions. The Curiosity rover system is the most current rover in operation on Mars, and has a number of notable features to enable it to traverse the challenging Martian terrain. Its rocker-bogie suspension system consists of six wheels arranged in such a way that enables a rocking motion between the front and back wheels. This design provides the platform with greater ability to traverse through uneven surfaces [1]. Furthermore, three primary modes of navigation were designed into the rover to enable effective navigation of difficult terrain. In blind-drive, human operators on Earth identify safe paths and send drive commands to the rover, thus requiring the lowest level of autonomy. In hazard avoidance mode, the rover autonomously chooses a path to follow such that hazards may be avoided, and is achieved through the use of images captured from body-mounted cameras. Finally, in visual odometry mode, the rover stops periodically to check for slippage by processing information from mast-mounted cameras. This mode may be used together with hazard avoidance at the expense of very low traversal speeds [22]. Indeed, it is evident that there exist serious drawbacks to both teleoperation-based and autonomous modes of navigation. The efficiency of blind-drive mode is hindered by the very long communication distances between human operator and the system, while autonomous navigation modes are slow due to the need to check for hazards and slippage frequently.

Recent attempts have been made to develop more effective solutions for planetary exploration. In [23], authors propose an enhanced system based on existing rover designs for planetary exploration. The proposed rover adopts the same rocker-bogie suspension mechanism from the Curiosity rover and additionally incorporates a mechanical arm and a humanoid robot carried onboard. These features provide the system with human-like investigation skills and extend the functionalities of the rover greatly. Robotic swarms have also been proposed for large-scale exploration [24]. These solutions revolve around the concept of a team of robots that work cooperatively to explore an environment, but acts as a single entity aiming to accomplish a common goal. This quickly introduces another kind of challenge: the coordination of multiple robots within an uncertain and unsafe environment. The authors in [25] discussed the benefits and challenges of multi-robot coordination from the perspective of planetary exploration. In their work, the appropriateness of reinforcement learning to overcome these challenges was also presented.

Onboard the international space station (ISS), Robonaut 2 has undergone extensive tests as a humanoid robot intended for routine maintenance and cleaning tasks. Programmed in the
Robot Operation System (ROS) framework, Robonaut 2 originally consisted of only a dexterous upper body with capabilities of operating systems inside the ISS. However, recently it has been equipped with a mobility platform that allows the robot to move around the ISS by gripping onto rails using manipulator legs [26]. The system is still a working development, but is progressing towards a fully-featured platform that will act as the foundation for future space robot missions.

\section{Search and Rescue}

Robotic systems deployed for search and rescue are typically required to perform a wide variety of tasks given the uncertainty of the environment in each instance of deployment. Ground vehicle robot systems are one type of robot that is commonly used to fulfill these requirements. The DARPA robotics competition provides a good illustration of this and aims to promote the development of RAS technology through a number of challenges. One particular robotic system developed for this competition is presented in [27]. Here a mobile manipulation robot, named Momaro, was designed to perform a number of tasks, including opening a door, turning a valve, cutting a hole into a piece of drywall, overcoming rough terrain and scattered debris and climbing stairs. The locomotion system consisted of a four 4 degrees-of-freedom (DOF) leg design with steerable wheels at each foot, providing omnidirectional movements and the capability to step over obstacles. The robot also possessed two 7 DOF arms used for manipulation purposes, and was tele-operated using a 3dimensional visualization system developed on the Oculus Rift. Furthermore, semi-autonomous control was used to perform stepping actions and weight shift handling.

The development of a ground vehicle robot for a different set of search and rescue needs was presented in [28]. The NuBot, which has been tested in the RoboCup Rescue Robot League competition, is a fully autonomous system designed for exploration purposes in an urban search and rescue setting. It is capable of performing simultaneous localization and mapping, victim detection, target selection, path planning and path following activities. For this particular system, a tracked platform with rotating sub-tracks was used as the means of locomotion in uneven terrain. The robot possesses a range of sensors, including LiDAR, thermal imager, microphone, inertial measurement unit (IMU) and a visual camera. Autonomy is achieved through automatic selection of a target point, from which a path is planned and executed using an onboard computer.

Aside from ground vehicle robots, UAVs have also become popular within search and rescue. This is largely because UAVs are agile and fast, possess good autonomous behavior, are low-cost to deploy, and have much more freedom of movement as they are not obstructed by obstacles on the ground. However, the use of UAVs also comes with a number of challenges. Some of these are: sensitivity to extreme weather conditions such as heavy winds; strict energy and weight limitations; difficulty in information exchange and 
coordination with other UAVs; and lower quality sensor data [29]. An example of UAV applications in search and rescue is provided in [30], where mountain rescue activities were discussed. The authors describe the use of fully autonomous UAVs to search for snow-covered survivors in the occurrence of avalanches in mountainous areas. This detection of survivors was achieved through the use of an avalanche beacon receiver equipped onto the UAV to communicate with avalanche beacon transmitters found on survivors. By managing information from onboard sensors, the UAV automatically adjusts its flying mission, thus reducing the tasks of the operator to monitoring activities only.

Search and rescue is one area of RAS applications where HRI is necessary. In many real-world scenarios, deployed robots must work cooperatively and collaboratively with human rescue teams in fast-changing and dynamic environments. The authors in [2] demonstrated one approach to this problem, where a gesture-based framework was tested in a simulated firefighting scenario to coordinate and command a team of robots. From their method, a Microsoft Kinect camera was used to recognize 12 gestures that provide specific control commands to the robots. This enabled quick and simple interactions between a human rescuer and the robots, providing a good integration between human cognitive decision making abilities and robotic capabilities. Testing in a simulated environment proved effective when considering darkness, smoke, crowds and users wearing firefighting uniforms.

\section{E. Others}

Many other application areas require the use of RAS technologies to assist with human activities due to the dangers posed by the associated working environments. The work presented in [31] discussed the implementation of a teleoperated mining robot for coal mining tasks. The system employs hydraulic power to drive a track-based platform and its excavation mechanism. Its onboard sensors include two visual cameras, two laser scanners, infrared sensors and proprioceptive sensors. Through data fusion techniques, the operator is provided with visual cues and pose information to aid tele-operation and supervisory tasks. The motivation behind the development of this system is the removal of humans from a harsh environment where the temperature and humidity is high, and where there is a large presence of harmful dusts and particles. Similarly, robots have been deployed for the inspection and maintenance of overhead power lines to remove personnel from the vicinity of high voltage exposures. An example of such robots is given in [32]. Here the design consisted of a wheeled robot with grippers to traverse across power lines with up to 30 degrees inclination and maneuver over vibration dampers. The system is powered by solar energy to provide long-term inspection possibilities, which is critical given the extensive lengths of transmission lines. Such a system proves highly beneficial for particularly hard to access and remote areas such as power lines in mountains and across large rivers. Indeed, other types of robots have been used for monitoring, inspection and surveillance of assets in the power industry [33]. These platforms are similar to those described for oil and gas applications and therefore will not be discussed further in this paper.

Robotic systems have also historically been exploited greatly to aid exploration for scientific purposes of locations previously inaccessible to mankind. The design and testing of a polar rover for Antarctic exploration is detailed in [34]. To overcome the naturally snowy surfaces of Polar Regions, triangular tracks were deployed to minimize the pressure applied on the ground, which consequently reduced the risk of sinkage. Furthermore, due to the rather featureless characteristic of these environments, a LiDAR system was installed to enable the generation of an environmental model for navigation purposes. Together with an integrated navigation system, which provides key information such as GPS coordinates, attitude and velocities, autonomy was achieved such that human intervention is primarily intended only for handling emergency situations and performing complex tasks.

Various other approaches to locomotion have been developed over the years for different types of harsh environments. The Djedi robot [35] was designed to climb a small, inclined airshaft within the Queen's chamber inside the Great Pyramid of Giza without inflicting damage to surrounding walls. The approach taken consisted of two pinion carriages arranged on a single rack. Linear actuators on each of these carriages alternately push against the wall to hold the robot in place as the other carriage advanced forward. [36] discussed the locomotion control behind a modular snake robot, which was composed of multiple identical modules connected by joints. This configuration offers greater flexibility and robustness to the navigation of rough terrain compared with classic wheel or track-base platforms. Similarly, legged robots, inspired by mammals from nature, have received much attention for navigating unstructured environments. [37] provides a brief history of this class of systems and describes the advancement of technology to effectively balance robots while walking, running or hopping. An interesting concept introduced in this paper is 'agile running', control techniques used to dynamically correct disturbances caused by issues such as slip, terrain uncertainty and unevenness.

In the medical field, development of in vivo robots have shown to effectively aid surgeons in minimally invasive surgery (MIS). The authors in [38] discussed the crucial aid offered by miniature robots in circumstances where big surgical robots are unavailable (such as for medical needs in space). Tele-operated fixed-base and mobile in vivo robots were tested to assist surgeons by providing wireless imaging on the abdominal cavity during surgery. Wheels on the mobile robot was designed such that it could maneuver across the abdominal organs without damaging local tissues. Impressively, even for a small-scale robot, on-board cameras may be adjusted to provide vision from various angles. A survey of continuum robots was conducted in [39] for broader applications in MIS. Here the authors defined a continuum robot as "an actuatable structure whose constitutive material forms curves with continuous tangent vectors". These robots effectively have infinite joints, enabling a high degree of 
flexibility in traversing very confined and complex spaces without damaging surrounding tissue walls. Developments in this area is largely ongoing, but opens up many possibilities in the world of medical healthcare.

\section{RESEARCH TRENDS AND DIRECTIONS}

This paper has provided an overview of RAS technologies deployed across a broad range of harsh environments. While the exact nature of these environments differ across applications, we have observed a number of key research trends common to the general theme of robotics for harsh environments.

Tethered robots have commonly appeared in a variety of applications to provide both power and information transmission. Yet this places certain levels of undesired restrictions on the robot. Development of untethered robots have been emerging, with a progressive shift towards the robots that can be deployed for the long periods. Existing longterm deployment solutions have involved either remote charging stations or the use of renewable energy (such as solar and wind) to recharge onboard batteries.

The wide variations in the properties of harsh environments have inspired many different designs for robot platforms. Aside from the traditional track or wheel based systems, UAVs have emerged as a very popular method to performing inspection in large-scale environments. The development of various types of locomotion systems have permitted more robust traversal of uneven and uncertain terrain, while humanoid robots provide more generalized human-like manipulation skills in places where humans cannot go. Indeed, humanoid robots are complex in nature, but they have begun to emerge as a viable and intelligent solution to several application areas, and will no doubt continue to be field-tested in a broader range of scenarios.

Swarm robotics have also seen widespread use across a number of areas and are suitable for applications where the presence of hazards to robots is high or where a large area must be explored. This concept of multi-robot teams extends beyond a swarm of identical robots. For complex tasks in challenging environments, it is becoming more common to see the cooperation between the robots designed for different purposes that collectively work towards a common goal. Nevertheless, there are many scenarios where humans will remain part of these teams on site. Hence the frameworks for effective HRI has garnered the equal attention in the advancement of RAS technologies.

In most applications discussed in this paper, we have discovered that a key task of the robot is to provide the visual information to a human operator about the local environment. Where possible, this is provided in the form of visual camera images. However, in cases where vision is unavailable due to such things as darkness, smoke or featureless landscapes, 3D visualization is achieved through environmental modelling using other sensory information provided by devices such as LiDAR systems and thermographic cameras. Additionally, sensors play a key role in enabling a system to perform other tasks. One major observation is the prominent use of teleoperation and semi-autonomous robots, but much fewer applications have relied upon fully-autonomous systems. A key issue stalling this advancement is the lack of proven reliability of full-autonomy in applications were mistakes or negligence can be catastrophic. For example, misidentifying a flaw in oil and gas structures could lead to a large-scale environmental disaster, while failure to avoid a serious hazard on the surface of Mars could mean the loss of a multi-billion pounds rover system that took years of manpower to develop. Indeed it has been observed that in applications where failure is more tolerable, autonomous systems have been deployed more frequently. This level of autonomy cannot be achieved without appropriate proprioceptive and exteroceptive sensors to enable a robot to understand its state and the state of the environment. Hence the development of sensor technologies has driven the possibilities of autonomy and will continue to do so in coming years. However, onboard data processing is an additional requirement for truly autonomous systems, which must be able to process the acquired sensory data to make appropriate decisions. Majority of deployed systems currently lack this capability, and still require the input of human operators to make cognitive decisions. The development of intelligent algorithms is an ongoing problem that is rapidly being explored. Much effort has been directed towards the application of soft computing techniques to produce novel decision making, task planning and control strategies. We therefore foresee much work in the future whereby autonomous and robust algorithms are integrated with robotic platforms for testing in real-world scenarios. Nevertheless, we expect human intervention to remain in the loop even with the advancement of full autonomy, due to the need for human knowledge and experience to handle emergencies and to execute the unusually complex tasks.

\section{Conclusions}

This paper has provided an overview of some key RAS technologies developed for application areas involving the interactions with harsh environments. Many of these environments are either too dangerous for humans to venture into, or are simply inaccessible. By exploiting the use of robots, humankind has been able to take a step forward in performing necessary activities in today's world. From fulfilling the world's demand for resources and providing safety and security to civilians through to advanced scientific discovery, RAS technology has undoubtedly become an essential asset that will continue to provide the various assistance in many tasks we previously could not accomplish.

\section{ACKNOWLEDGMENT}

This research is funded by the Engineering and Physical Sciences Research Council (EPSRC) under its Doctoral Training Partnership Programme (DTP 2016-2017 University of Strathclyde, Glasgow, UK). 


\section{REFERENCES}

[1] J. P. Grotzinger, J. Crisp, A. R. Vasavada, R. C. Anderson, C. J. Baker, R. Barry et al., "Mars science laboratory mission and science investigation," Space Sci. Rev., vol. 170, no. 1-4, pp. 5-56, Sep. 2012.

[2] F. De Cillis, G. Oliva, F. Pascucci, R. Setola, and M. Tesei, "On field gesture-based human-robot interface for emergency responders," in 2013 IEEE International Symposium on Safety, Security, and Rescue Robotics, SSRR 2013, 2013.

[3] J. P. H. Steele, Q. Han, H. Karki, K. Al-Wahedi, A. A. Ayoade, M. R. Sweatt et al., "Development of an oil and gas refinery inspection robot," in Volume 4A: Dynamics, Vibration, and Control, 2014, p. V04AT04A016.

[4] R. S. Freitas, M. F. S. Xaud, I. Marcovistz, A. F. Neves, R. O. Faria, G. P. S. Carvalho et al., "The embedded electronics and software of DORIS offshore robot," IFAC-PapersOnLine, vol. 48, no. 6, pp. 208-213, 2015.

[5] A. Shukla and H. Karki, "Application of robotics in onshore oil and gas industry-A review Part I," Rob. Auton. Syst., vol. 75, pp. 490-507, 2016.

[6] Y. Wu, A. Noel, D. D. Kim, K. Youcef-Toumi, and R. Ben-Mansour, "Design of a maneuverable swimming robot for in-pipe missions," in 2015 IEEE/RSJ International Conference on Intelligent Robots and Systems (IROS), 2015, pp. 4864-4871.

[7] W. Z. Khan, M. Y. Aalsalem, W. Gharibi, and Q. Arshad, "Oil and Gas monitoring using Wireless Sensor Networks: Requirements, issues and challenges," in 2016 International Conference on Radar, Antenna, Microwave, Electronics, and Telecommunications (ICRAMET), 2016, pp. 31-35.

[8] D. Wu, D. Chatzigeorgiou, K. Youcef-Toumi, and R. Ben-Mansour, "Node localization in robotic sensor networks for pipeline inspection," IEEE Trans. Ind. Informatics, vol. 12, no. 2, pp. 809-819, Apr. 2016.

[9] F. Mazzini and S. Dubowsky, "An experimental validation of robotic tactile mapping in harsh environments such as deep sea oil well sites," Springer Berlin Heidelberg, 2014, pp. 557-570.

[10] W. Fischer, F. Tache, and R. Siegwart, "Inspection system for very thin and fragile surfaces, based on a pair of wall climbing robots with magnetic wheels," in 2007 IEEE/RSJ International Conference on Intelligent Robots and Systems, 2007, pp. 1216-1221.

[11] D. English, "Improving assetmanagement at process plants through UAV (unmanned aerial vehicle) inspections," in Abu Dhabi International Petroleum Exhibition and Conference, 2015.

[12] A. Shukla, H. Xiaoqian, and H. Karki, "Autonomous tracking of oil and gas pipelines by an unmanned aerial vehicle," in 2016 IEEE 59th International Midwest Symposium on Circuits and Systems (MWSCAS), 2016, pp. 1-4.

[13] Ø. Ødegård, A. J. Sørensen, R. E. Hansen, and M. Ludvigsen, “A new method for underwater archaeological surveying using sensors and unmanned platforms," IFAC-PapersOnLine, vol. 49, no. 23, 2016.

[14] L. D. L. Barker and L. L. Whitcomb, "Preliminary survey of underwater robotic vehicle design and navigation for under-ice operations," in IEEE International Conference on Intelligent Robots and Systems, 2016, vol. 2016-Novem.

[15] Y. Yang, S. Hirose, P. Debenest, M. Guarnieri, N. Izumi, and K. Suzumori, "Development of a stable localized visual inspection system for underwater structures," Adv. Robot., vol. 30, no. 21, 2016.

[16] H. Yoshida, S. Ishibashi, O. Yutaka, M. Sugesawa, and K. Tanaka, "A concept design of underwater docking robot and development of its fundamental technologies," in Autonomous Underwater Vehicles 2016, AUV 2016, 2016.

[17] A. Matos, A. Martins, A. Dias, B. Ferreira, J. M. Almeida, H. Ferreira et al., "Multiple robot operations for maritime search and rescue in euRathlon 2015 competition," in OCEANS 2016 - Shanghai, 2016.

[18] T. Tanaka, T. Matsuo, H. Fuji, and T. Takimoto, "Development of quadrotor type underwater robot for fixed-point observation," in Autonomous Underwater Vehicles 2016, AUV 2016, 2016.

[19] O. Khatib, X. Yeh, G. Brantner, B. Soe, B. Kim, S. Ganguly et al., "Ocean one: A robotic avatar for oceanic discovery," IEEE Robot.
Autom. Mag., vol. 23, no. 4, 2016.

[20] K. Yoshida, "Achievements in space robotics," IEEE Robot. Autom. Mag., vol. 16, no. 4, pp. 20-28, Dec. 2009.

[21] R. P. Mueller and P. J. van Susante, "A review of Eextra-terrestrial mining robot concepts," in Earth and Space 2012, 2012, pp. 295-314.

[22] L. Ding, H. Gao, Z. Deng, Y. Li, G. Liu, H. Yang, and H. Yu, "Threelayer intelligence of planetary exploration wheeled mobile robots: Robint, virtint, and humint," Sci. China Technol. Sci., vol. 58, no. 8, pp. 1299-1317, Aug. 2015.

[23] S. Aswath, N. Ajithkumar, C. K. Tilak, N. Saboo, A. Suresh, R Kamalapuram et al., An intelligent rover design integrated with humanoid robot for alien planet exploration, vol. 345. 2015.

[24] E. Staudinger, S. Zhang, A. Dammann, and C. Zhu, "Towards a radiobased swarm navigation system on mars Key technologies and performance assessment," in 2014 IEEE International Conference on Wireless for Space and Extreme Environments (WiSEE), 2014, pp. 1-7.

[25] L. Yliniemi, A. K. Agogino, and K. Tumer, "Multirobot coordination for space exploration," AI Mag., vol. 35, no. 4, 2014.

[26] J. Badger, D. Gooding, K. Ensley, K. Hambuchen, and A. Thackston, ROS in space: A case study on robonaut 2, vol. 625. 2016.

[27] M. Schwarz, T. Rodehutskors, D. Droeschel, M. Beul, M. Schreiber, N. Araslanov et al., "NimbRo rescue: Solving disaster-response tasks with the mobile manipulation robot Momaro," J. F. Robot., vol. 34, no. 2 , 2017.

[28] Y. Liu, Y. Zhong, X. Chen, P. Wang, H. Lu, J. Xiao, and H. Zhang, "The design of a fully autonomous robot system for Urban search and rescue," in 2016 IEEE International Conference on Information and Automation, IEEE ICIA 2016, 2017.

[29] S. Waharte and N. Trigoni, "Supporting search and rescue operations with UAVs," in 2010 International Conference on Emerging Security Technologies, 2010, pp. 142-147.

[30] M. Silvagni, A. Tonoli, E. Zenerino, and M. Chiaberge, "Multipurpose UAV for search and rescue operations in mountain avalanche events," Geomatics, Nat. Hazards Risk, pp. 1-16, Oct. 2016.

[31] S. Huh, U. Lee, H. Shim, J.-B. Park, and J.-H. Noh, "Development of an unmanned coal mining robot and a tele-operation system," in International Conference on Control, Automation and Systems, 2011.

[32] G. Zhao, L. Wang, S. Cheng, and J. Zhang, "Design and realization of a mobile robot for power transmission lines inspection," in 2011 IEEE International Conference on Mechatronics and Automation, ICMA 2011, 2011.

[33] L. Li, D. Li, Y. Li, B. Zhang, J. Zhao, C. Zhang, and Z. Dai, "A state-ofThe-Art survey of the robotics applied for the power industry in China," in 2016 4th International Conference on Applied Robotics for the Power Industry, CARPI 2016, 2016.

[34] C. Chen, C. Bu, Y. He, and J. Han, "Design, implementation and experimental tests of a new generation of Antarctic rover," in 2012 IEEE International Conference on Robotics and Biomimetics (ROBIO), 2012, pp. 2144-2149.

[35] R. Richardson, S. Whitehead, T. Ng, Z. Hawass, A. Pickering, S. Rhodes, R. Grieve, et al, "The 'Djedi' robot exploration of the southern shaft of the queen's chamber in the Great Pyramid of Giza, Egypt," J. F. Robot., vol. 30, no. 3, pp. 323-348, May 2013.

[36] X. Ye, Y. Niu, H. Wang, and T. Meng, "Locomotion control for a modular snake robot over rough terrain," in 2010 2nd Conference on Environmental Science and Information Application Technology, ESIAT 2010, 2010, vol. 2.

[37] J. Bhatti, A. R. Plummer, P. Iravani, and B. DIng, "A survey of dynamic robot legged locomotion," in Proceedings of 2015 International Conference on Fluid Power and Mechatronics, FPM 2015, 2015.

[38] M. E. Rentschler, S. R. Platt, K. R. Berg, J. Dumpert, D. R. Oleynikov, and S. M. Farritor, "Miniature in vivo robots for remote and harsh environments," IEEE Trans. Inf. Technol. Biomed., vol. 12, no. 1, pp. 66-75, Jan. 2008.

[39] J. Burgner-Kahrs, D. C. Rucker, and H. Choset, "Continuum robots for medical applications: A survey," IEEE Trans. Robot., vol. 31, no. 6, pp. 1261-1280, Dec. 2015. 